\title{
Effect of Cadmium exposure in the ubiquitous coccolithophore Emiliania huxleyi
}

Amorim, A.***, F. Africano***, V. Brotas***, M.L. Dâmaso-Rodrigues *, V. Veloso*, A.P. Alves de Matos****,*****, M.F. Caeiro********, R. Costa $* * * * * * * * * *$, P.A. Carvalho******, M. Cachão ***

*Centro de Oceanografia, Faculdade de Ciências da Universidade Lisboa (FCUL), 1749-016 Lisboa, Portugal

**Departamento de Biologia Vegetal, FCUL, 1749-016 Lisboa, Portugal

***Centro de Geologia e Departamento de Geologia, FCUL, 1749-016 Lisboa, Portugal

**** Anatomia Patológica, Centro Hospitalar de Lisboa Central - HCC, Rua da Beneficência 8, 1069-166 Lisboa, Portugal and Centro de Investigação Interdisciplinar Egas Moniz (CiiEM), Quinta da Granja , Monte de Caparica, 2829-511 Caparica, Portugal

***** Centro de Estudos do Ambiente e do Mar (CESAM), FCUL, 1749-016 Lisboa, Portugal

******ICEMS, Instituto Superior Técnico, Universidade de Lisboa, Av. Rovisco Pais, 1049-001 Lisboa, Portugal

The coccolithophores are a marine phytoplankton group that can play an important role in selective uptake of Cadmium (Cd) in neritic environments. Under project Cd-ToxCoN (PTDC/MAR/102800/2008) studies were conducted to investigate the in vitro reaction of Emiliania huxleyi to Cd exposure, namely changes in the crystal lattice of (cocco)liths, the calcite elements of the exoskeleton (coccosphere).

Uni-algal cultures of E. huxleyi were obtained by multiple cell isolation from the same water sample collected from Portuguese offshore waters, during opportunity cruises (Portuguese Marine Navy). Several morphotypes of E. huxleyi are currently recognized. The identification of the morphotype of E. huxleyi from Portuguese coastal waters was done by morphological analysis of the exoskeleton using scanning electron microcopy (JEOL JSM5200LV and FEG-SEM JEOL 7001F) and by DNA sequencing of tufA gene following [1].

Experiments were conducted in batch cultures grown in enriched sea-water medium $(\mathrm{K} / 10)$ under constant environmental conditions ( $14 \mathrm{~h} \mathrm{~L}: 10 \mathrm{~h} \mathrm{D}, 15^{\circ} \mathrm{C}, 40 \mu \mathrm{mol}$ photons $\mathrm{m}^{-2} \mathrm{~s}^{-1}$ ). At the exponential growth phase, 3 replicate E. huxleyi cultures were subject for $48 \mathrm{~h}$ to $\mathrm{Cd}$ concentrations of $10 \mu \mathrm{gL}^{-1}$ and $100 \mu \mathrm{gL}^{-1}$, two and three orders of magnitude above average marine concentration values respectively. In each case three additional replicate cultures with no $\mathrm{Cd}$ added were used as control.

The effect of $\mathrm{Cd}$ was evaluated by measuring in vivo fluorescence (ratio of variable $\left(\mathrm{F}_{\mathrm{v}}\right)$ to maximum $\left(\mathrm{F}_{\mathrm{m}}\right)$ fluorescence) (Water PAM fluorometer - Walz) and by detailed morphometric analysis of coccospheres and liths performed on SEM micrographs. The parameters measured are those presented in Figure 1. Malformed, damaged or tilted liths were not measured.

Morphological observations of the coccosphere and liths and DNA sequencing allowed the identification of the isolated strains of E. huxleyi as morphotype A. Results on the Cd exposure experiments indicate that E. huxleyi type A seems to tolerate high concentrations of $\mathrm{Cd}$. Despite the very high concentration of $\mathrm{Cd}$ tested no lethal limit was reached and $\mathrm{F}_{\mathrm{v}} / \mathrm{F}_{\mathrm{m}}$ values recorded after $48 \mathrm{~h}$ at $10 \mu \mathrm{gL}^{-1} \mathrm{Cd}(0.607 \pm 0.008)$ and $100 \mu \mathrm{gL}^{-1} \mathrm{Cd}$ $(0.603 \pm 0.008)$, very similar to the values recorded in the control cultures $(0.642 \pm 0.016$ and $0.636 \pm 0.018$, respectively) and before $\mathrm{Cd}$ addition $(0.642 \pm 0.012$ and $0.636 \pm 0.007$, respectively).

Regarding the different morphometric parameters analysed on coccospheres and liths, no significant measurable effects were observed. However, we observed, in response to increasing Cd concentration, an increasing number of liths with fused or partially fused elements (Fig. 2) suggesting a higher calcification of liths. This interpretation is supported by the results of the analysis of coccosphere calcium content. Cultures exposed to Cd presented a higher $\mathrm{Ca}$ content compared to control cultures. The highest values were recorded in coccospheres submitted to $100 \mu \mathrm{g} . \mathrm{L}^{-1}$ of $\mathrm{Cd}$ representing a $65 \%$ increase in Ca content in comparison to control coccospheres.

E. huxleyi is ubiquitous in present day oceans and usually very tolerant to culture conditions and is thus frequently used as a model species in the study of coccolithophores. These same characteristics may also justify the observed high tolerance to $\mathrm{Cd}$. Studies with other species are needed to clarify if the surprising resistance to $\mathrm{Cd}$ is unique to $E$. huxleyi or characteristic of other unicellular algae with calcium carbonate shells. 
References

1. Cook S.S. et al., J. Phycol., 47:615-626, 2011.

This work was funded by projects PEst-OE/Mar/UI0199/2011, Pest/OE/CTE/UI/0263 and PTDC/MAR/102800/2008

a)

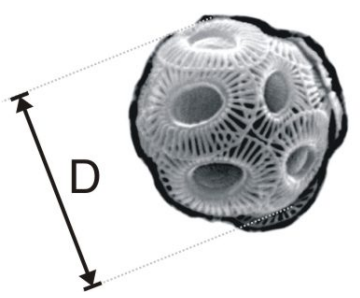

b)

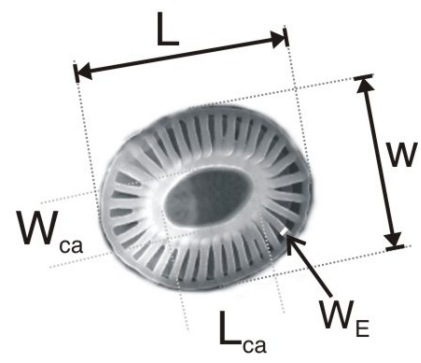

Figure1. Morphometric parameters measured on E. huxleyi coccosphere (a) and coccolith (b). D - coccosphere diameter; L - length of the coccolith; W - width of the coccolith; Lca - length of the central area; Wca - width of the central area; $\mathrm{W}_{\mathrm{E}}-$ width of the element (crystalith) of the distal shield.
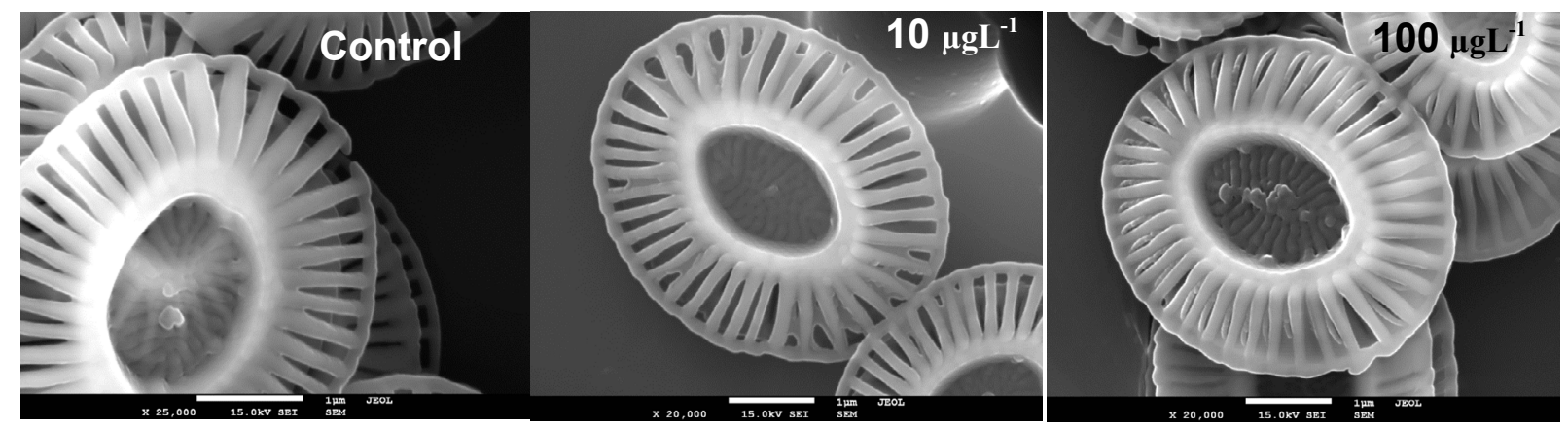

Figure 2. Coccoliths of E. huxleyi type A from cultures not subjected to $\mathrm{Cd}$ (Control) and cultures subjected to 10 and $100 \mu \mathrm{gL}^{-1}$. Note the number of fused elements in the distal shield of the coccolith. 